\title{
COMPARACIÓN DE LA CINEMÁTICA DE LOS MIEMBROS DE DOS ESPECIES DE ROEDORES HISTRICOGNATOS (Cuniculus taczanowskii y Dinomys branickii)
}

\section{COMPARISON OF THE LIMB'S KINEMATIC OF TWO HYSTRICOGNATH RODENT SPECIES (Cuniculus taczanowskii and Dinomys branickii)}

\author{
Karin Osbahr ${ }^{1}$, José Luis Azumendi ${ }^{2}$ \\ ${ }^{1}$ Líder Grupo de Investigación en Fauna Silvestre. Universidad de Ciencias Aplicadas y Ambientales U.D.C.A. Dirección para \\ correspondencia: Calle 222 No. 55-37 Bogotá - D.C kosbahr@udca.edu.co, ${ }^{2}$ Director científico Fundación Colombiana de \\ Estudios de Parásitos FUNCEP funcepdircientifico@cable.net.co
}

Rev. U.D.C.A Act. E Div. Cient. 12 (2): 39-50, 2009

\section{RESUMEN}

La cuantificación de la cinemática de los miembros provee información de los aspectos comportamentales de la locomoción. Las diferencias morfológicas conllevan a diferencias en la capacidad de desempeño, lo cual, a su vez, resulta en diferencias en el uso de hábitat. El estudio comparativo de los movimientos de los miembros anterior y posterior de dos especies de roedores histricomorfos permite reconocer los principios básicos de la locomoción de Cuniculus taczanowskii y Dinomys branickii. Para registrar el movimiento de ambas especies, se utilizaron fotogramas, midiendo los ángulos durante las fases de apoyo y de balanceo. La comparación de los diferentes segmentos durante el desplazamiento resalta las partes proximales, como la escápula y el fémur, para producir la mayor parte de la propulsión de ambos miembros. La diferencia en la amplitud del ángulo de las articulaciones, así como la duración del momento de contacto con el suelo, contribuye en la velocidad, en la amplitud y en la fuerza del desplazamiento de estas especies, lo que les permite hacer un uso diferencial del hábitat disponible.

Palabras clave: Cinemática, esqueleto apendicular, C. taczanowskii, D. branickii.

\section{SUMMARY}

Quantifying the limb kinematics provide information on the locomotor behavior. Morphological differences lead to distinctiveness in the performance ability which in turn results in a diversification of habitat use. The comparative study of the fore and hind limb movements of two species of rodents is useful to recognize the basic principles of the locomotion of Cuniculus taczanowskii and Dinomys branickii. To record the movements of both species photograms were used on which the angles were measured through the stance and swing phase. The comparison of different segments during the movement highlights the scapula and femur to produce most of the propulsion force in both species. The difference in amplitude of the joint angle and the length of time contact with the soil, contributes to the speed, breadth and strength of the movement of these species which allows them to make a differential use of the available habitat.

Key words: Kinematics, appendicular skeleton, C. taczanowskii, D. branickii. 


\section{INTRODUCCIÓN}

En la conformación del esqueleto apendicular de los mamíferos se conjugan la relevancia ecológica de la locomoción y el comportamiento locomotor requerido, para lograr el mejor desempeño en las funciones críticas para la supervivencia y la reproducción, tales como la búsqueda de alimento, la evasión de depredadores y las interacciones sociales, propias de cada especie (Reilly et al. 2007). Debido a esto, el estudio de la morfología funcional de las extremidades en movimiento es altamente complejo. Durante el movimiento locomotor debe existir un equilibrio entre el esfuerzo que implica la locomoción y el gasto energético involucrado al moverse a diferentes velocidades (Taylor et al. 1982). En la mayoría de los casos, los animales se movilizan a velocidades óptimas reduciendo el costo energético de la locomoción (Hildebrand E Goslow, 2004), regulando así la disponibilidad de energía para otras actividades, entre ellas, la reproducción. Esta regulación de energía, se encuentra relacionada con el patrón de movimiento de las extremidades durante la locomoción, el cual, puede variar de acuerdo a la velocidad, al terreno y a la necesidad de maniobrar. Cada tipo de marcha equivale a una velocidad óptima, en la que se consume un mínimo de calorías por metro (Hildebrand, 1966; Perry et al. 1988; Alexander, 1989). Las extremidades se conforman de tal forma que son funcionalmente óptimas no solo para desplazarse, minimizando el costo energético, sino también para soportar la masa corporal (Christiansen, 2002).

Desde un punto de vista mecánico, la organización del cuerpo de los cuadrúpedos es esencialmente segmentada, flexible y soportada por cuatro miembros diferenciados en tres segmentos unidos por puntos articulares, que corresponden a las articulaciones (Rocha-Barbosa et al. 2005). Aún cuando durante la locomoción interactúan los diferentes componentes anatómicos, las extremidades representan uno de los elementos más importantes. La variación en su diseño es importante desde el punto de vista mecánico, debido a que afecta las fuerzas que deben ejercer los músculos para soportar la extremidad, durante el contacto con el sustrato (Biewener, 1989). Los músculos soportan y mueven al animal de acuerdo al momento o fuerza de torsión relativa a cada articulación dentro de la extremidad. Durante la locomoción cada segmento de los miembros rota con respecto a las articulaciones que lo componen, donde los ángulos de éstas varían, adaptándose a un cambio cíclico durante las diferentes fases que conforman un paso (Jenkins, 1971; Brinkman, 1981; Reilly \& DeLancey, 1977a,b; Irschick \& Jayne, 1999). El movimiento del miembro, ya sea anterior o posterior de un animal, está dividido en una fase de apoyo (E) en la que se encuentran retraídos y una fase de balanceo $(F)$, donde los miembros se extienden (Philippson, 1905; Fischer et al. 2002; Rocha-Barbosa et al. 2005). Dado que los movimientos de los segmentos son exclusivamente monofásicos, los de las articulaciones pueden llegar a ser bifásicos, incluyendo dos extensiones y dos flexiones por paso. Los movimientos de las articulaciones varían de acuerdo a la especie (Schilling, 2005), que para el caso de los roedores Caviomorfos, se expresa en una gran diversidad de tipos de locomoción, reflejando una acción recíproca entre las influencias funcionales y evolutivas del esqueleto apendicular (Weisbecker $\mathcal{E}$ Schmid, 2007). Adicionalmente, se ha considerado que las condiciones en las que un cuadrúpedo puede usar un miembro para un determinado propósito en particular, así como para el soporte y propulsión del cuerpo, dependen de las posiciones de las cuatro extremidades, relativas al centro de gravedad del cuerpo (Gray, 1943; Witte et al. 2002).

La mayoría de los mamíferos utilizan durante la locomoción cuadrúpeda marchas con secuencias laterales, donde éste patrón se caracteriza por que en un ciclo, dado el apoyo del miembro anterior, sigue, inmediatamente, después del miembro posterior ipsolateral. Al caminar, el patrón de movimiento de las extremidades es simétrico y cada uno debe completar un ciclo de igual longitud y duración, iniciando y finalizando en la fase de apoyo. Aún cuando especies diversas aplican las mismas secuencias, la distribución en el tiempo de las diferentes combinaciones, para apoyar los miembros, no es la misma (Hildebrand, 1966; 1989).

Estudiar la mecánica de la locomoción es un paso importante para comprender el comportamiento y las adaptaciones de las distintas especies. En el caso concreto de los roedores, la comprensión de la locomoción se ha centrado en especies de pequeño tamaño corporal, siendo poco estudiadas las especies plantígradas y digitígradas, comprendidas entre 1 a $20 \mathrm{~kg}$ del suborden Hystrocognatha, el cual, a su vez, incluye a los caviomorfos suramericanos. Estudios generales realizados con este grupo (Rocha-Barbosa et al. 1996; 2005; Elissamburu E Vizcaíno, 2004; 
Weisbecker \& Schmid, 2007; Reilly et al. 2007) reportan variaciones en las estrategias relacionadas con los costos locomotores y han descrito algunas similitudes morfológicas y tendencias evolutivas en la locomoción, que permiten relacionar a los Cavioidea con los ungulados.

Las distintas adaptaciones de un organismo, como la capacidad de obtener alimento y la dinámica de la locomoción, están relacionadas con su estructura corporal. Así, es posible suponer que la anatomía, como también los ángulos entre cada uno de los huesos que componen los miembros de un animal, permiten identificar parámetros de desplazamiento (Reilly et al. 2007). Por lo tanto, el propósito del presente trabajo, fue el de realizar una aproximación desde la cinemática del miembro anterior y posterior de Cuniculus taczanowskii y de Dinomys branickii, con el fin de comprender sus patrones de desplazamiento y las diferencias en el comportamiento locomotor, observadas durante el desplazamiento exploratorio del entorno. Estas especies son de hábitos nocturnos y habitan en ambientes escarpados de difícil acceso, cubiertos con vegetación propia del bosque andino y páramo (Osbahr, 1995). Se conoce que presentan diferencias en la longitud de los segmentos óseos del esqueleto apendicular (Osbahr et al. 2009), además de registrar diferencias morfológicas y comportamentales (Tabla 1). El estudio de las características del desplazamiento aporta información para interpretar el uso de hábitat y los rangos de acción de cada una de estas especies de roedores, que coexisten en el mismo hábitat.

Tabla 1. Características morfológicas y comportamentales de las especies en estudio.

\begin{tabular}{|c|c|c|c|c|}
\hline & & Dinomys branickii & Cuniculus taczanowskii & Fuente \\
\hline \multirow{5}{*}{$\begin{array}{c}\text { MORFOLOGÍA } \\
\text { (promedios, en } \\
\mathrm{mm} \text { ) }\end{array}$} & Largo total & 645 & 610 & \multirow{5}{*}{$\begin{array}{l}\text { Osbahr } \\
\text { et al. } 2009\end{array}$} \\
\hline & Largo cola & 178 & 9 & \\
\hline & $\begin{array}{l}\text { Miembro anterior con } \\
\text { uña }\end{array}$ & 68 & 56 & \\
\hline & $\begin{array}{l}\text { Miembro posterior con } \\
\text { uña }\end{array}$ & 112 & 92 & \\
\hline & Peso (kg) & 10 a 15 & 5 a 7 & \\
\hline \multirow{4}{*}{ ANATOMÍA } & Miembro anterior & 4 dedos, $\mathrm{V}$ vestigial & \multirow{2}{*}{5 dedos, I y $\vee$ reducidos } & \multirow{2}{*}{ Nowak, 1991} \\
\hline & Miembro posterior & 5 dedos & & \\
\hline & $\begin{array}{l}\text { Longitud total miembro } \\
\text { anterior (promedio } \mathrm{mm} \text { ) }\end{array}$ & 204,91 & 143,94 & \multirow{2}{*}{ Osbahr et al. 2009} \\
\hline & $\begin{array}{l}\text { Longitud total miembro } \\
\text { posterior (promedio mm) }\end{array}$ & 244,62 & 195,05 & \\
\hline \multirow{8}{*}{ HÁBITOS } & \multirow{4}{*}{ Locomoción } & plantígrado & digitígrado & Nowak, 1991 \\
\hline & & cursorial & fosorial & Osbahr et al. 2009 \\
\hline & & escansorial & terrestre & $\begin{array}{l}\text { Wilson \& Reeder, } \\
2005\end{array}$ \\
\hline & & capacidad para trepar & capacidad para saltar & \multirow{5}{*}{$\begin{array}{l}\text { López et al. 2000; } \\
\text { Osbahr, } 2003\end{array}$} \\
\hline & \multirow{4}{*}{ Comportamiento } & grupos familiares & solitario & \\
\hline & & & utiliza sendas & \\
\hline & & $\begin{array}{l}\text { manipula alimento, } \\
\text { posición sentada }\end{array}$ & transporta alimento & \\
\hline & & $\begin{array}{c}\text { bípedo durante } \\
\text { encuentros agresivos }\end{array}$ & & \\
\hline
\end{tabular}




\section{MATERIALES Y MÉTODOS}

El estudio se realizó con dos hembras adultas de $D$. branickii y de $C$. taczanowskii alojadas en un encierro mixto de $25 \times 10 \mathrm{~m}$, ambientado de forma natural con cuevas, suelo en tierra y pasto kikuyo (Kikuyuochloa clandestina), ubicado en la Unidad de Investigación en Fauna Silvestre de la Universidad de Ciencias Aplicadas y Ambientales U.D.C.A.

Para registrar el movimiento de las extremidades, se llevaron a cabo grabaciones, con una cámara SONY Handycam Vision modelo CCD - TRV25. Aprovechando los períodos diurnos de mayor actividad de cada especie (López et al. 2000), se filmaron los animales durante una hora por 15 días consecutivos, previa ubicación de la cámara, en un plano paralelo al piso. Luego, se editaron los videos por medio del programa Combustion ${ }^{\circledR} 2008$ seleccionando 215 fotogramas, de 640 pixeles, equivalentes a 22,58cm que registraran, a una velocidad de 25 fotogramas/ segundo, cuatro ciclos consecutivos de la secuencia de movimientos realizados durante las fases de apoyo y balanceo en cada especie mientras se desplazaba sobre un mismo sustrato y en la misma dirección. Sobre estas secuencias, se determinó el diagrama de la marcha y se calculó el factor de rendimiento expresado, como el porcentaje de una zancada, en el que cada extremidad se encuentra en fase de apoyo (Hildebrand, 1966). Los fotogramas obtenidos, se analizaron en un editor de imágenes (Adobe ${ }^{\circledR}$ Photoshop ${ }^{\circledR}$ CS4), para obtener mediciones de la altura a la que cada especie eleva cada extremidad sobre el sustrato, durante la fase de balanceo. Para esta medida, se tuvo en cuenta el borde distal de los metatarsos y de los metacarpos, respectivamente. Dado que la velocidad de locomoción influye en los patrones cinemáticos de las extremidades, se asumió lo que se conoce como "velocidad preferida" durante la marcha, es decir, el momento en el que cada pie está en contacto con el suelo más de la mitad del tiempo durante una pisada (Hildebrand, 1966, 1985; Hoyt $E$ Taylor, 1981).

Debido a que estudios realizados por Pike $E$ Alexander (2002) en animales domésticos y de zoológico demostraron que no existe discrepancia entre los valores de los ángulos en animales marcados previamente en los puntos de intersección articular frente a animales sin marcar, se definieron, mediante imágenes radiológicas, las uniones articulares de las extremidades sobre ejemplares adultos completos, depositados en líquido en la colección biológica de la U.D.C.A (registro IAvH 53), estableciendo, como puntos de referencia de las articulaciones, las líneas dorsales con manchas blancas, que identifican ambas especies. Por tal razón, ninguno de los ejemplares vivos fue rasurado para ayudar a la identificación de los puntos articulares.

Sobre los fotogramas y empleando como referencia las imágenes radiológicas obtenidas, se trazaronlíneas, cuyos vértices definen los cuatro puntos de inserción articular en el miembro anterior y en el posterior (Rocha-Barbosa et al. 2005) que, en su conjunto, permiten establecer la cinemática para cada especie (Pike E Alexander, 2002); los ángulos, se midieron utilizando un transportador de $360^{\circ}$. Los resultados obtenidos, se analizaron utilizando estadística descriptiva (Sokal \& Rohlf, 2000).

\section{RESULTADOS Y DISCUSIÓN}

Al caminar, tanto C. taczanowskii como D. branickii, registran una marcha simétrica con ocho momentos de apoyo o combinaciones de soporte específicos, en los que se alternan secuencias laterales y diagonales, antes de comenzar un nuevo ciclo (Figura 1). En ambas especies, las secuencias laterales permiten mayor estabilidad (Gray, 1943) a la vez que se evita la interferencia del miembro posterior con el anterior, en los momentos en los que las extremidades de un mismo lado del cuerpo actúan como pares laterales, en la fase de balanceo (Hildebrand, 1966). Sin embargo, al analizar el diagrama de la marcha, se halló que, en el caso de C. taczanowskii, el factor de rendimiento es menor, tanto para el miembro posterior $(63,3 \%)$ como para el anterior $(23,3 \%)$, mientras que $D$. branickii registra valores mayores para ambos miembros (70,58\% y 35,29\%, respectivamente). De acuerdo con Candela E Picasso (2008), la diferencia inter e intraespecífica en los factores de rendimiento, se correlaciona con la longitud de la pata y de la mano y aún cuando esta variable no equivale a una medida directa de la velocidad de la locomoción (Hildebrand, 1966) permite inferir que al caminar C. taczanowskii se desplaza a mayor velocidad que $D$. branickii, por lo cual, finaliza un ciclo en menor tiempo (Figura 2). La diferencia en los factores de rendimiento entre ambas especies es explicada igualmente por el hecho que, en $D$. branickii la longitud total de los miembros es mayor que en C. taczanowskii (Tabla 1). La posición 

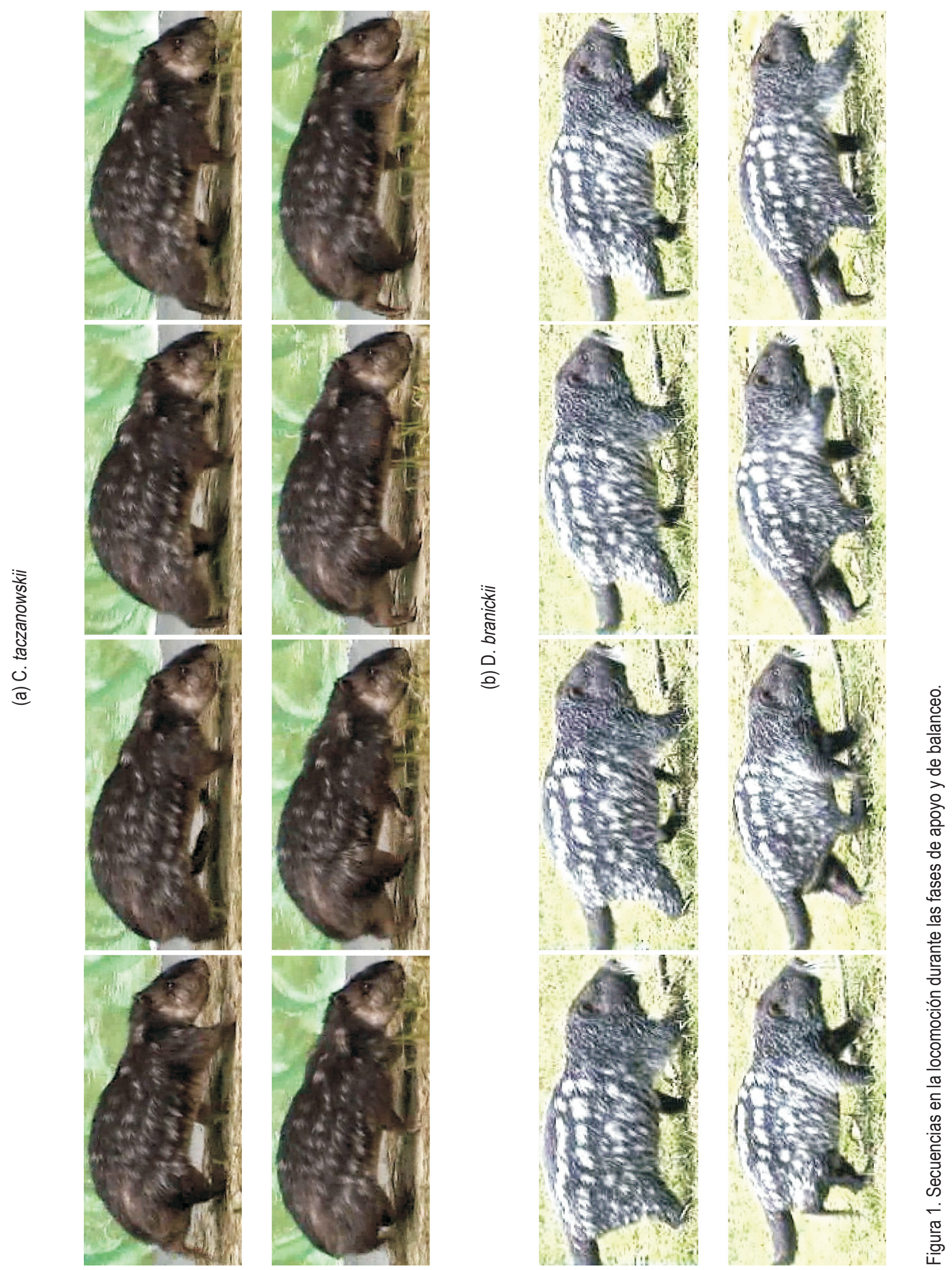
(a) C. taczanowskii

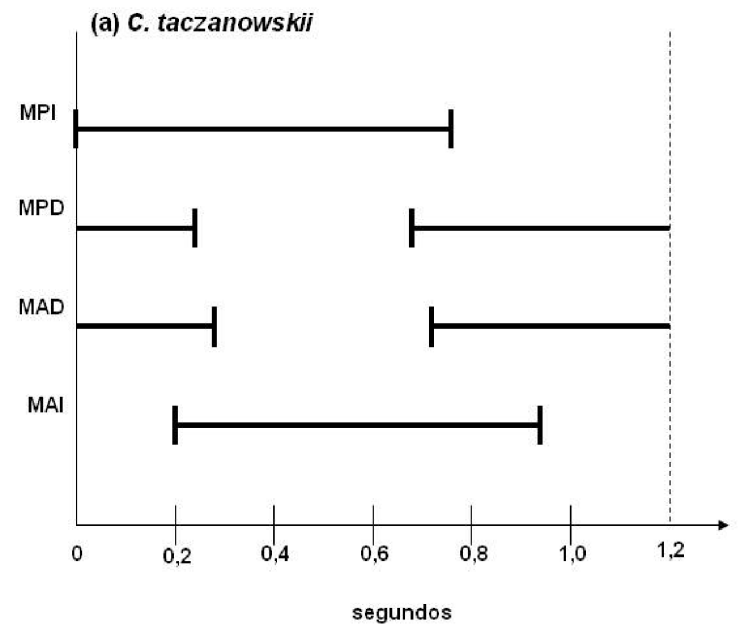

(b) D. branickii

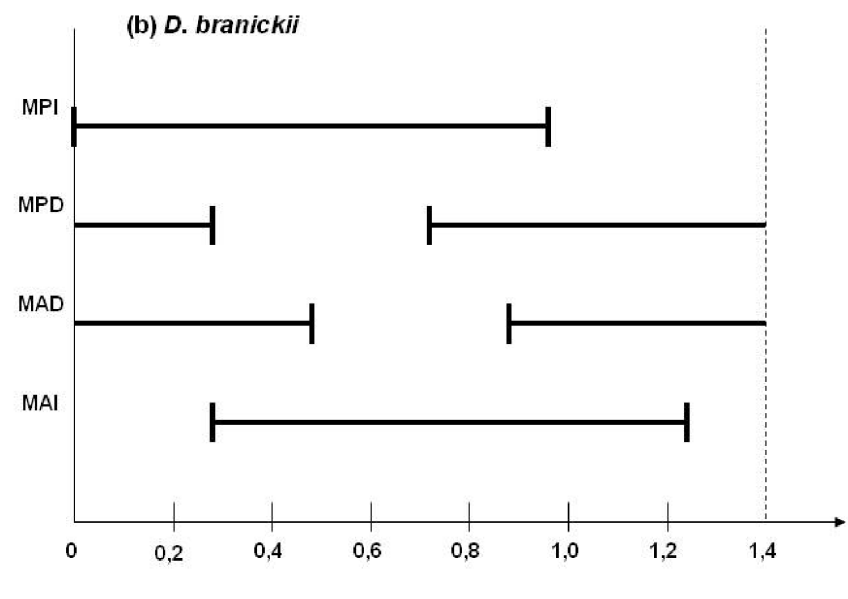

segundos

Figura 2. Diagramas de la marcha representando un ciclo, teniendo como referencia el miembro posterior izquierdo (MPI). Las barras representan la fase de apoyo de cada extremidad. MPD = miembro posterior derecho; MAD = Miembro anterior derecho; MAI = miembro anterior izquierdo.

del centro de gravedad influye a su vez en los factores de rendimiento (Hildebrand, 1989). D. branickii al ser un animal más robusto obtiene una mayor estabilidad en los momentos de apoyo, debido a que el triángulo de soporte, formado por dos miembros anteriores y un miembro posterior, se mantiene durante un período más prolongado en cada paso. A su vez, las extremidades más largas junto con su condición plantígrada y la cola larga (Tabla 1), compensan en términos de estabilidad con respecto al centro de gravedad del cuerpo, los períodos más prolongados, en los que los miembros se encuentran en fase de balanceo.
Al avanzar las dos especies registran diferencias en la altura a la que se elevan las extremidades con respecto al sustrato, durante las fases de balanceo. C. taczanowskii eleva en menor proporción cada extremidad (altura máxima promedio C.t. $(\mathrm{n}=56)=5,53 \pm 1,64 \mathrm{~cm} ; D . b$. $(n=40)=7,59 \pm 2,04 \mathrm{~cm})$; sin embargo, ambas especies presentan una mayor altura en los miembros anteriores con respecto a los posteriores (Figura 3). Las mediciones obtenidas a partir de los fotogramas permitieron establecer, igualmente, que durante una zancada $C$. taczanowskii avanza en promedio $17,38 \pm 2,89 \mathrm{~cm}$, mientras que $D$. branickii recorre una distancia

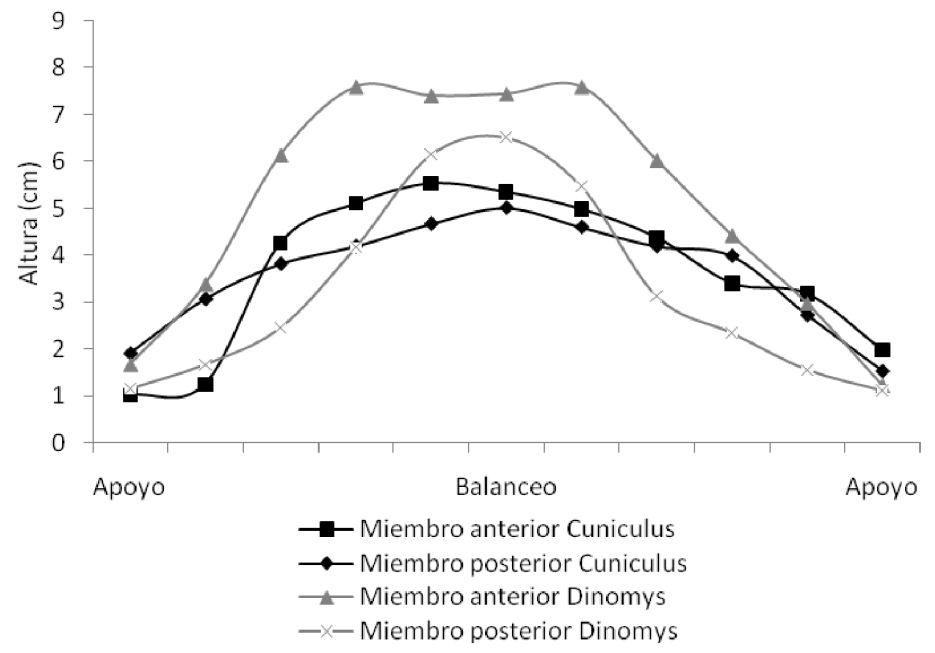

Figura 3. Altura promedio a lo largo de un paso de las extremidades con respecto al sustrato durante la fase de balanceo, en cada secuencia de la locomoción. 
promedio mayor $(20,73 \pm 1,18 \mathrm{~cm})$. Por lo tanto, la velocidad estimada al caminar equivale a $0,98 \mathrm{~km} / \mathrm{h}$ por hora, para C. taczanowskii (1paso=0,64s) y $1,04 \mathrm{~km} / \mathrm{h}$, para $D$. branickii (1paso $=0,72 \mathrm{~s}$ ). Al avanzar realiza un movimiento lateral del brazo, característico durante la fase de balanceo, lo que aumenta el impulso, lo cual, se refleja en una mayor amplitud en la pisada. C. taczanowskii avanza con los miembros en posición sagital, que le permite aumentar la frecuencia de las pisadas y, por lo tanto, la velocidad durante el desplazamiento.

En general, la longitud de la pisada depende del recorrido angular, la altura del centro de rotación y de la longitud de la extremidad, dada por la sumatoria de los segmentos que la componen. El movimiento del miembro anterior es ejecutado, principalmente, por el desplazamiento de la escápula y el húmero, mientras que para el miembro posterior, se hace referencia a la horizontal del ilion y del fémur (Fischer et al. 2002). Debido a que los cuadrúpedos comparten una serie de patrones comunes en su mecánica locomotora (Demes et al. 1994), en las especies en estudio, la variación angular en las articulaciones analizadas, muestra igualmente las menores amplitudes durante un ciclo en el miembro anterior (horizontal de la escápula) y en la articulación equivalente del miembro posterior (horizontal del íleon), mientras que las articulaciones conformadas por los huesos largos de cada miembro (escápula-humeral, húmero-radial, ilio-femoral, femorotibial, respectivamente) presentan amplitudes mayores. La articulación de la muñeca (radio-metacarpal), en ambas especies, registra valores promedio superiores a los $100^{\circ}$, derivados, especialmente, de la flexión de ésta articulación, durante la fase de balanceo (Figura 4). Al analizar en detalle los resultados obtenidos para las angulaciones de las articulaciones del miembro anterior durante un paso (Figura 5 y 6), los ángulos articulares del hombro registran valores promedio superiores en D. branickii (Figura 5) (apoyo=116,83 $\pm 7,28$; balanceo $=128,36 \pm 19,20 ; \quad n=12) \quad y$ menores en C. taczanowskii (Figura 6) (apoyo=88,28 $\pm 9,69$; balanceo $=100,00 \pm 23,15)$. De igual manera, en el miembro posterior la articulación de la cadera presenta valores promedio mayores en $D$. branickii $($ apoyo $=113,42 \pm 7,02 ; \quad$ balanceo $=103,30 \pm 8,65) \quad$ y menores en C. taczanowskii (apoyo $=91,00 \pm 7,45$; balanceo $=71,60 \pm 13,12$ ).

(a) C. taczanowskii $(\mathrm{n}=30)$
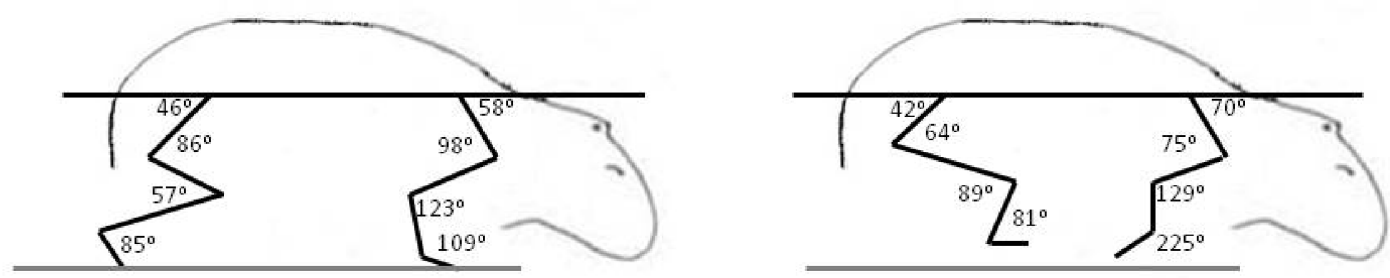

(b) D. branickii $(\mathrm{n}=35)$

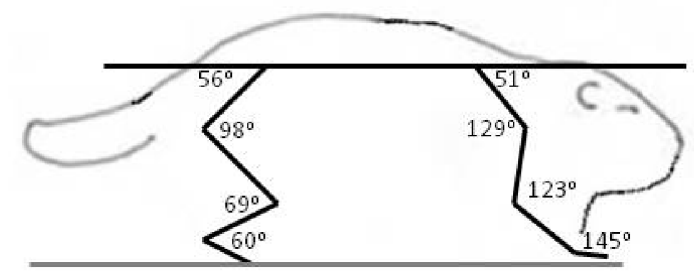

Fase de apoyo

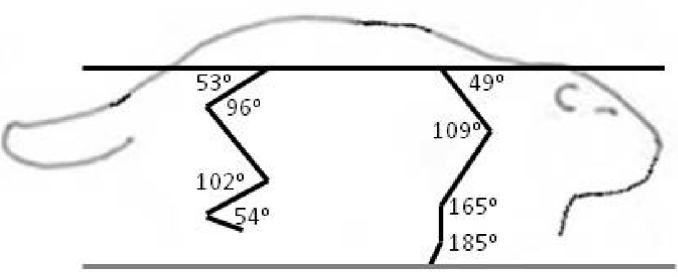

Fase de balanceo

Figura 4. Amplitudes angulares promedio de las articulaciones del miembro anterior y posterior, durante la locomoción de las especies en estudio. 
(a) Miembro anterior

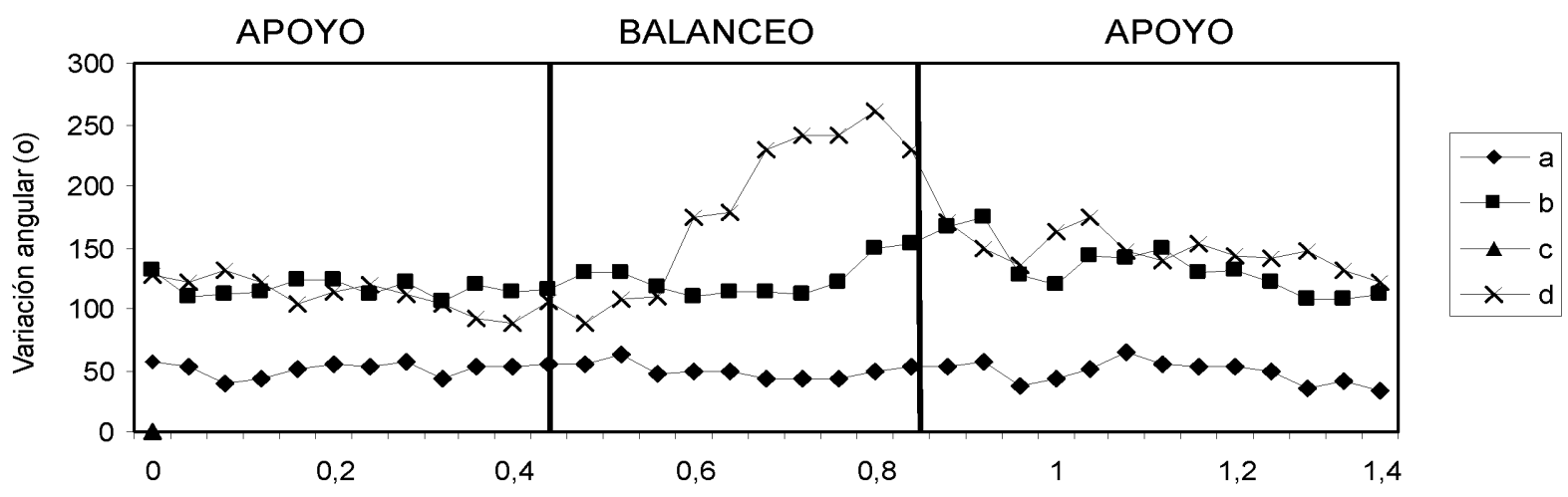

(b) Miembro posterior

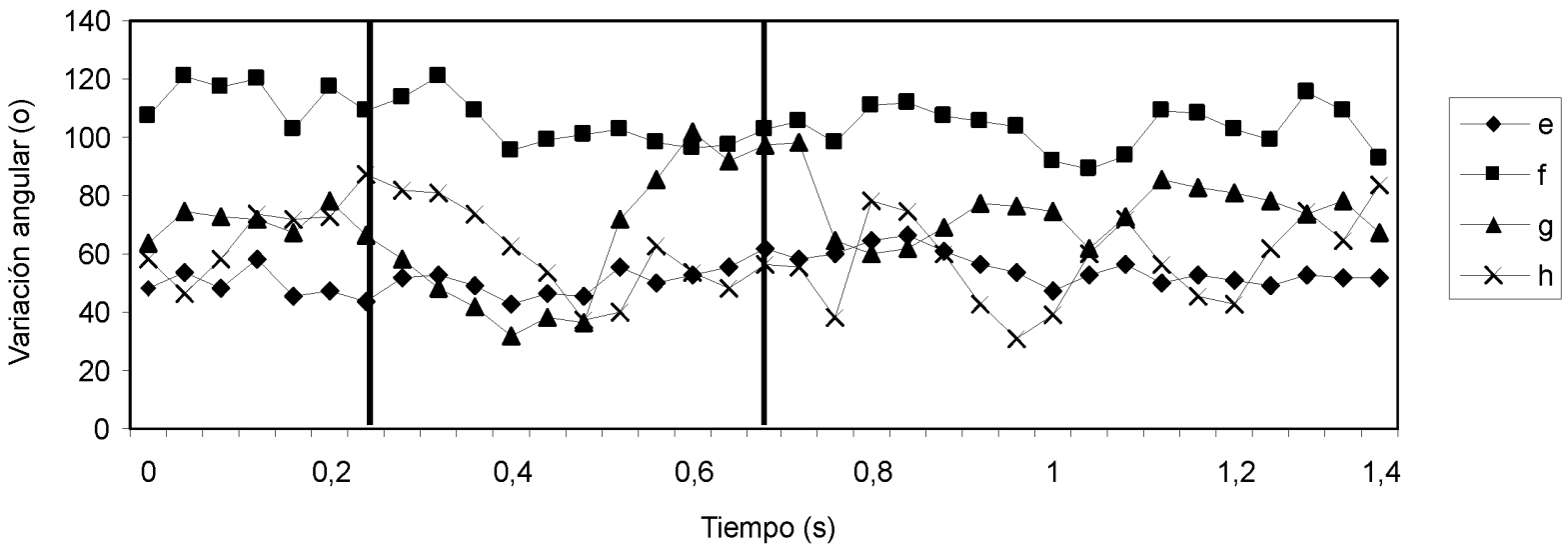

a) Horizontal de la escápula; b) Articulación escápulo - humeral (hombro); c) Articulación húmero - radial (codo); d) Articulación radio - carpiana (muñeca); e) Horizontal del llion; f) Articulación coxo - femoral (cadera); g) Articulación tibio - femoral (rodilla);

h) Articulación tibio - tarsiana (tobillo).

Figura 5. Patrón de la variación angular de las articulaciones en las fases de apoyo y balanceo, durante un ciclo de la locomoción en $D$. branickii.

En el caso de C. taczanowskii, las extremidades más cortas y el desplazamiento digitígrado le permiten desarrollar el trote para moverse a mayor velocidad, aumentando la eficiencia durante la locomoción y realizar desplazamientos por medio de saltos largos o altos. Cuando las articulaciones alcanzan un alto grado de flexión durante la fase de apoyo, los ángulos más cerrados generan la posibilidad de almacenar una mayor cantidad de energía, que puede ser transformada en energía elástica (Alexander, 1989; 2002; Rocha-Barbosa et al. 2005), la cual, puede funcionar a manera de resorte, facilitando este tipo de locomoción, cuando el animal se siente amenazado. Adicionalmente, existe una relación entre el almacenamiento de esta energía elástica en combinación con la duración de la fase de apoyo, donde a menor tiempo de contacto con el suelo aumenta el almacenamiento de energía, lo que permite un desplazamiento a mayores velocidades (Cavagna et al. 1964). D. branickii igualmente utiliza los saltos cortos como mecanismo de huída (López et al. 2000), pero a diferencia de $C$. taczanowskii, no se desplaza al trote para aumentar la velocidad de desplazamiento, sino que acelera el movimiento anadeante, típico de la especie. Se podría inferir que, en este caso, la capacidad de salto es una ventaja esporádica similar a la de C. taczanowskii; cada especie la emplea de acuerdo a sus adaptaciones y sus características morfofisiológicas (Witte et al. 2002). De acuerdo a lo anterior es posible deducir que las 
(a) Miembro anterior

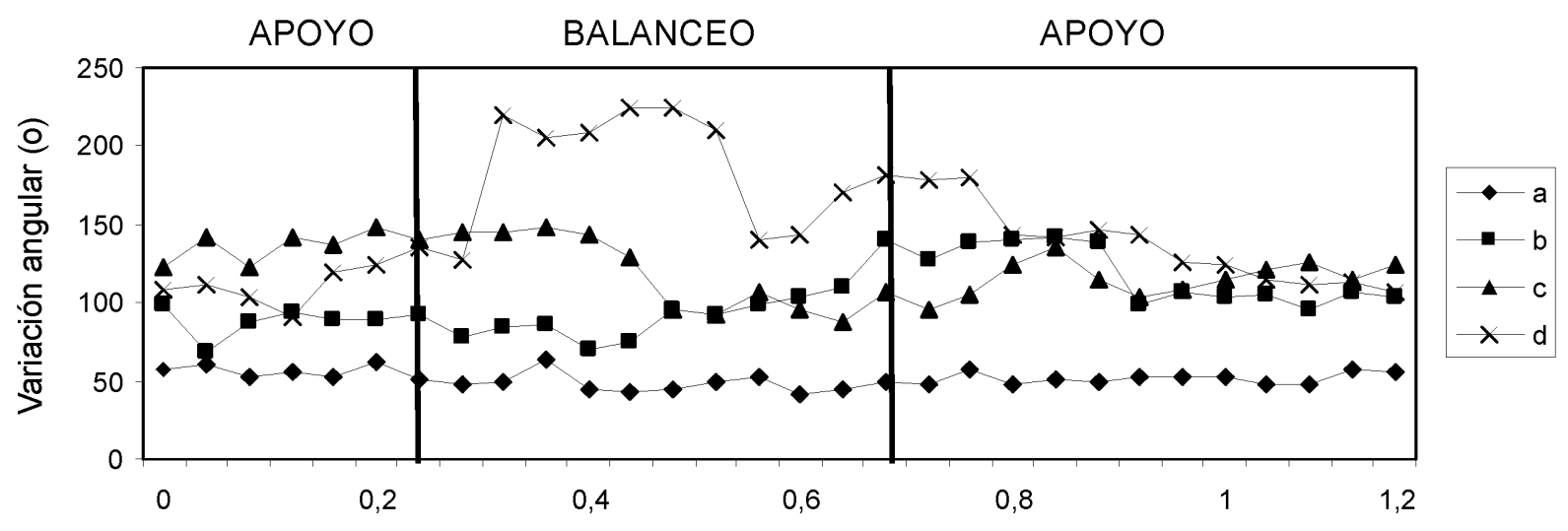

(b) Miembro posterior

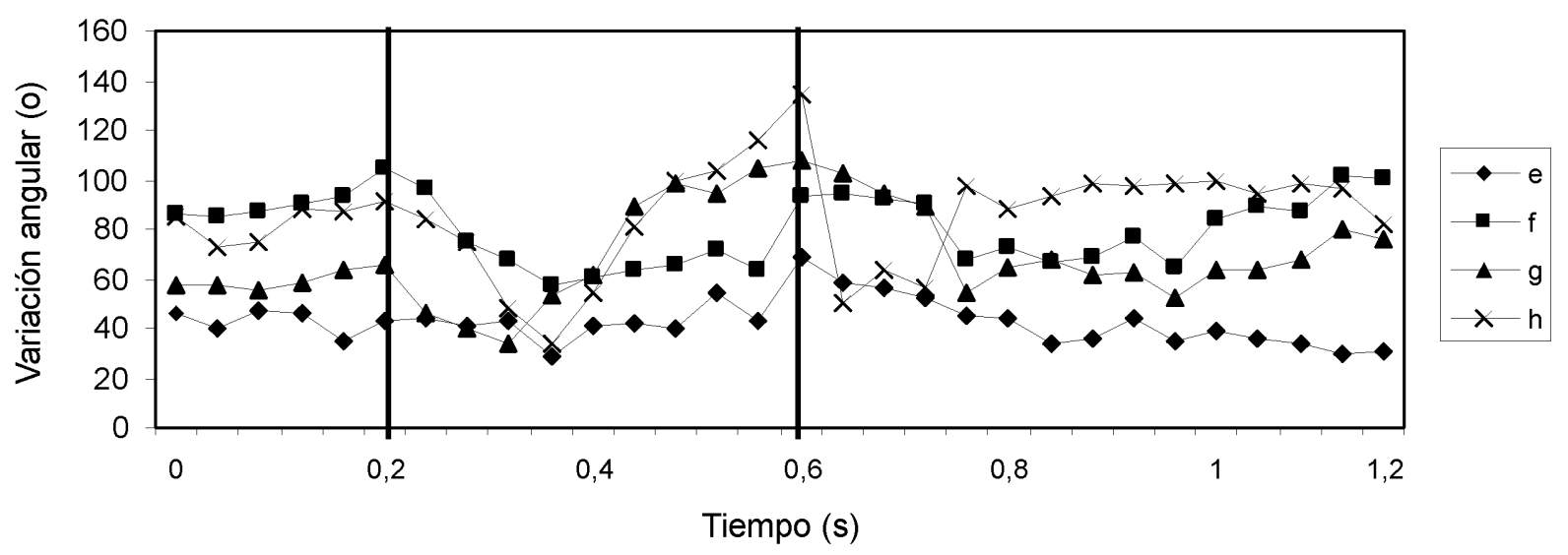

Figura 6. Patrón de la variación angular de las articulaciones en las fases de apoyo y balanceo, durante un ciclo de la locomoción en C. taczanowskii. Para comprensión de la nomenclatura ver leyenda figura 5.

angulaciones más abiertas registradas para $D$. branickii explican la mayor amplitud de paso que, a su vez, le permiten recorrer distancias más extensas durante las exploraciones nocturnas en búsqueda de alimento o refugio. Por otro lado, las angulaciones más cerradas en C. taczanowskii, le pueden permitir reservar una mayor cantidad de energía, lo cual, podría ser ventajoso en el momento de modificar el tipo de desplazamiento, empleando una mayor velocidad, es decir, que como mecanismo de evasión ante los depredadores está acondicionado para la huída, mientras que $D$. branickii encara al depredador, haciendo uso de sus fuertes incisivos.
Aún cuando los animales en general se desplazan por diferentes razones, cabe mencionar que los desplazamientos dentro de un área de acción se encuentran relacionados con la frecuencia de forrajeo y los patrones de alimentación. Es así como se puede sugerir que siendo $D$. branickii una especie grupal, que se destaca por inspeccionar los parches de alimentación y que manipula los alimentos con los miembros anteriores en posición sentada (López et al. 2000; Osbahr E Restrepo-Mejía, 2001), invierte menos tiempo en la búsqueda de alimento y, por tanto, se puede desplazar más lento que $C$. taczanowskii, donde siendo una especie solitaria, transporta el alimento para consumirlo en lugares protegidos (Parra E Osbahr, 
2000), es decir, que de acuerdo a lo sugerido por Garland (1983), el costo ecológico de la locomoción, definido en términos del porcentaje del gasto energético diario dedicado al desplazamiento, es menor para $D$. branickii. A su vez, el modelo analizado por Carbone et al. (2005) indica que los grupos taxonómicos difieren en la distancia diaria de desplazamiento, respecto a su masa corporal y a sus hábitos forrajeros, lo que respalda las diferencias encontradas para las dos especies, al ser D. branickii más robusta y, por ende, tener una mayor masa corporal (Tabla 1).

Es importante indicar que las características mencionadas anteriormente pueden ser expresadas por cada especie en diferente grado y, a su vez, son adaptaciones desarrolladas de acuerdo al hábitat en que se desempeñe. Muchas especies de roedores terrestres se mueven sobre troncos verticales o utilizan ramas o árboles caídos en el sotobosque (Gallardo-Santis et al. 2005), adaptando su comportamiento locomotor, de acuerdo a la inclinación del sustrato (Lammers \& Biknevicius, 2004; Lammers et al. 2006). Cuando los cuadrúpedos terrestres se desplazan sobre sustratos arbóreos, los miembros anteriores y posteriores generan diferentes puntos de rotación con respecto al eje longitudinal de la rama (Lammers, 2007). Aún, cuando D. branickii claramente es un animal trepador (Osbahr, 1998), también C. taczanowskii utiliza para desplazarse las ramas retorcidas de la vegetación, propia de los bosques andinos, en búsqueda de retoños especialmente de las plantas de la familia Ericaceae (Osbahr, 2003), por lo tanto, que las angulaciones articulares reportadas en este trabajo, respaldan la posibilidad que las dos especies se puedan desenvolver sobre superficies inclinadas, modificando la marcha para desplazarse sobre sustratos arbóreos reduciendo, especialmente, la velocidad. Las vertientes pendientes y rocosas que conforman el hábitat natural de ambas especies (Osbahr, 1995) implican que con frecuencia los animales se deben desplazar sobre estas superficies, para lo cual, deben modificar las fuerzas de soporte sobre los miembros. Haciendo uso de las angulaciones más cerradas $C$. taczanowskii supera los obstáculos por medio del salto, mientras que $D$. branickii trepa sobre ellos, modificando las fuerzas que deben soportar los miembros, de acuerdo al desplazamiento cuesta arriba o abajo.

Se concluye, que al caminar las especies utilizan la marcha simétrica, pero se diferencian en el factor de rendimiento y en la amplitud del paso, por lo que se desplazan a velocidades diferentes. Considerando que la capacidad locomotora se encuentra estrechamente asociada a la historia natural de los animales, las diferencias observadas en las amplitudes de los ángulos de las articulaciones del miembro anterior y posterior entre las dos especies indican, igualmente, habilidades diferentes en estas especies para moverse en su hábitat natural y, por lo tanto, se puede asociar a diferencias en el acceso a componentes distintos, durante el aprovechamiento del espacio.

AGRADECIMIENTOS: Los autores agradecen a la ZGAP (Zoologische Gesellschaft fuer Arten und Populationsschutz), a la ZooAG Bielefeld, al Tierpark Cottbus, a los Zoológicos de Newquay, Chester y de Los Angeles, por el permanente apoyo recibido durante el desarrollo del presente trabajo. Igualmente, agradecen la colaboración recibida por parte de los Médicos Veterinarios Pedro Acevedo, Andrea Villamizar y Daniela Espinosa. Conflicto de intereses: Hacemos constar que el manuscrito fue preparado y revisado con la participación de los dos autores, quienes declaramos que no existe ningún conflicto de intereses que ponga en riesgo la validez de los resultados presentados.

\section{BIBLIOGRAFÍA}

1. ALEXANDER, R. McN. 1989. Elastic mechanisms in animal movement. Cambridge: Cambridge University Press. 180p.

2. ALEXANDER, R. McN. 2002. Tendon elasticity and muscle function. Comp. Biochen. Physiol. A Comp. Physiol. 133:1001-1011.

3. BIEWENER, A. 1989. Mammalian terrestrial locomotion and size. Bioscience. 39(11):776-783.

4. BRINKMAN, D. 1981. The hind limb step cycle of Iguana and primitive reptiles. J. Zoology Lond. 181:91-103.

5. CANDELA, A.M.; PICASSO, M.B.J. 2008. Functional anatomy of the limbs of Erethizontidae (Rodentia, Caviomorpha): Indicators of locomotor behavior in miocene porcupines. J. Morphology. 269:552593. 
6. CARBONE, C.; COWLISHAW, G.; ISAAC, N.J.B.; ROWCLIFF, J.M. 2005. How far do animals go? Determinants of day range in mammals. The American Naturalist. 165(2):290-297.

7. CAVAGNA, G.A.; SAIBENE, F. P.; MARGARIA, R. 1964. Mechanical work in running. J. Appl. Physiology. 19:249-256.

8. CHRISTIANSEN, P. 2002. Locomotion in terrestrial mammals: the influence of body mass, limb length and bone proportions on speed. Zoological J. Linnean Soc. 136:685-714.

9. DEMES, B.; LARSON, S.G.; STERN, J.T.; JUNGERS, W.L.; BIKNEVICIUS, A.R.; SCHMITT, D. 1994. The kinetics of primate quadrupedalism: "hind limb drive' reconsidered. J. Hum. Evol. 26:353-374.

10. ELISSAMBURU, A.; VIZCAÍNO, S.F. 2004. Limb proportions and adaptations in caviomorph rodents (Rodentia: caviomorpha). J. Zoology Lond. 262:145-159.

11. FISCHER, M.S.; SCHILLING, N.; SCHMIDT, M.; HAARHAUS, D.; WITTE, H. 2002. Basic limb kinematics of small therian mammals. J. Exp. Biology. 205:1315-1338.

12. GALLARDO-SANTIS, A.; SIMONETTI, J.A.; VASQUEZ, R.A. 2005. Influence of tree diameter on climbing ability of small mammals. J. Mammal. 86: 969-973.

13. GARLAND, T. 1983. Scaling the ecological cost of transport to body mass in terrestrial mammals. The American Naturalist. 121(4):571-587.

14. GRAY, J. 1943. Studies in the mechanics of the tetrapod skeleton. University of Cambridge. p.88-116.

15. HILDEBRAND, M. 1966. Analysis of the symmetrical gaits of tetrapods. Folia Biotheoretica. 6:9-22.

16. HILDEBRAND, M. 1985. Walking and running. In: Hildebrand, M.; Bramble, D.M.; Llem, K.F.; Wake, D.B. eds. Functional Vertebral Morphology, Belknap Press p.38-57.

17. HILDEBRAND, M. 1989. The quadrupedal gaits of vertebrates. Bioscience. 39(11):766-776.
18. HILDEBRAND, M.; GOSLOW, G.E. 2004. Vergleichende und funktionelle Anatomie der Wirbeltiere. Springer Verlag. 709p.

19. HOYT, D.F.; TAYLOR, C.R. 1981. Gait and the energetics of locomotion in horses. Nature. Lond. 292:239-240.

20. IRSCHICK, D.J.; JAYNE, B.C. 1999. Comparative three-dimensional kinematics of the hindlimb for high-speed bipedal and quadrupedal locomotion of lizards. J. Exp. Biology. 202:1047-1065.

21. JENKINS, F.A. Jr. 1971. Limb posture and locomotion in the Virginia opossum (Didelphis marsupialis) and in other noncursorial mammals. J. Zoology Lond. 165:303-315.

22. LAMMERS, A.R. 2007. Locomotor kinetics on sloped arboreal and terrestrial substrates in a small quadrupedal mammal. Zoology. 110:93-103.

23. LAMMERS, A.R.; BIKNEVICIUS, A.R. 2004. The biodynamics of arboreal locomotion: the effects of substrate diameter on locomotor kinetics in the gray short-tailed opossum (Monodelphis domestica). J. Exp. Biology. 207:4325-4336.

24. LAMMERS, A.R.; EARLS, K.D.; BIKNEVICIUS, A.R., 2006. Locomotor kinetics and kinematics on inclines and declines in the gray short-tailed opossum Monodelphis domestica. J. Exp. Biol. 209:4154-4166.

25. LÓPEZ, L.; LÓPEZ, I.; MORA J.; OSBAHR, K. 2000. Estudio preliminar del comportamiento de $D i$ nomys branickii (Peters, 1873) en cautiverio. Rev. U.D.C.A Act. E Div. Cient. 3(1):28-35.

26. NOWAK, R.M. 1991. Walker's mammals of the world. John Hopkins Univ. Press. 5th ed. Baltimore, Maryland, U.S.A. p.812-815.

27. OSBAHR, K. 1995. Distribución geográfica de tres especies de roedores histricomorfos en la región andina de Colombia. Informe técnico. Programa de becas para la conservación. WCI, FES, GEA. 120 p.

28. OSBAHR, K. 1998. Guía para la conservación de la "guagua loba" o "pacarana" (Dinomys branickii). 
$1^{a}$ ed. SECAB, Ciencia y Tecnología (Bernal, H.Y.; Farfán M, eds.). Convenio Andrés Bello. Bogotá. 65:1-43.

29. OSBAHR, K.; RESTREPO-MEJÍA, D. 2001. Determinación de calcio, hierro, proteína y otros requerimientos de nutrientes de Dinomys branickii (Peters 1873). Rev. U.D.C.A Act.E Div. Cient. 4(1):44-55.

30. OSBAHR, K. 2003. Patrones de distribución del recurso alimenticio disponible para Agouti taczanowskii (Rodentia, Agoutidae) en un fragmento de bosque andino nublado. Rev. U.D.C.A Act. E Div. Cient. 6(1):38-49.

31. OSBAHR, K.; ACEVEDO, P.; VILLAMIZAR, A.; ESPINOSA, D. 2009. Comparación de la estructura y de la función de los miembros anterior y posterior de Cuniculus taczanowskii y Dinomys branickii. Rev. U.D.C.A Act. E Div. Cient. 6(1):37-51.

32. PARRA, A.; OSBAHR, K. 2000. Contribución al conocimiento anatómico del aparato digestivo del tinajo de páramo (Agouti taczanowskii, Stolzman 1865). Rev. U.D.C.A. Act.E Div. Cient. 3(1):21-27.

33. PERRY, A.K.; BLICKHAN, R.; BIEWENER, A.; HEGLUND, N.C.; TAYLOR, R. 1988. Preferred speeds in terrestrial vertebrates: are they equivalent? J. Exp. Biology. 13:207-219.

34. PHILIPPSON, M. 1905. L' autonomie et la centralisation dans le systéme nerveux des animaux. Trav. Lab. Physiol. Solvay (Bruxelles). 7:1-208.

35. PIKE, A.V.L.; ALEXANDER, R. McN. 2002. The relationship between limb-segment proportions and joint kinematics for the hind limbs of quadrupedal mammals. J. Zoology Lond. 258:427-433.

36. REILLY, S.M.; DeLANCEY, M.J. 1997a. Sprawling locomotion in the lizard Sceloporus clarkia: quantitative kinematics of a walking trot. J. Exp. Biology. 200:753-765.

37. REILLY, S.M.; DeLANCEY, M.J. 1997b. Sprawling locomotion in the lizard Sceloporus clarkia: the effects of speed on gait, hindlimb kinematics, and axial bending during walking. J. Zoology Lond. 243:417-433.
38. REILLY, S.M.; MCELROY, E.J.; BIKNEVICIUS, A.R. 2007. Posture, gait and the ecological relevance of locomotor costs and energy-saving mechanisms in tetrapods. Zoology. 110:271-289.

39. ROCHA-BARBOSA, O.; RENOUS, S.; GASC, J. P. 1996. Comparison of the fore and hindlimb kinematics in the symmetrical and asymmetrical gaits of a caviomorph rodent, the domestic guinea pig Cavia porcellus (Linné, 1758) (Rodentia: Caviidae). Ann. Sci. Nat. (Zool. Biol. Anim). 17(4):149-165.

40. ROCHA-BARBOSA, O.; FIUZA DE CASTRO, M.; RENOUS, S.; GASC, J. P. 2005. Limb joint kinematics and their relation to increase speed in the guinea pig Cavia porcellus (Mammalia: Rodentia). J. Zoology. Lond. 266:293-305.

41. SCHILLING, N. 2005. Ontogenetic development of locomotion in small mammals - a kinematic study. J. Exp. Biology. 208:4013-4034.

42. SOKAL, R.R.; ROHLF, J. 2000. Biometry. Principles and Practices of Statistics in Biological Research. $3^{\mathrm{a}} \mathrm{Ed}$. Freeman and Company. New York (USA). 887p.

43. TAYLOR, C.R.; HEGLUND, N.C.; MALOY, G.M.O. 1982. Energetics and mechanics of terrestrial locomotion. J. Exp. Biology. 97:1-21.

44. WEISBECKER, V.; SCHMID, S. 2007. Autopodial skeletal diversity in hystricognath rodents: Functional and phylogenetic aspects. Mammalian Biology. 72(1):27-44.

45. WILSON, D.E.; REEDER, D.A. 2005. Mammal Species of the World. A Taxonomic and Geographic Reference (3rd ed), Johns Hopkins University Press, 2142p.

46. WITTE, H.; BILTZINGER, J.; HACKERT, R.; SCHILING, N.; SCHMIDT, M.; REICH, C.; FISCHER, M. S. 2002. Torque patterns of the limbs of small therian mammals during locomotion on flat ground. J. Exp. Biology. 205:1339-1353.

Recibido: Febrero 10 de 2009

Aceptado: Noviembre 20 de 2009 